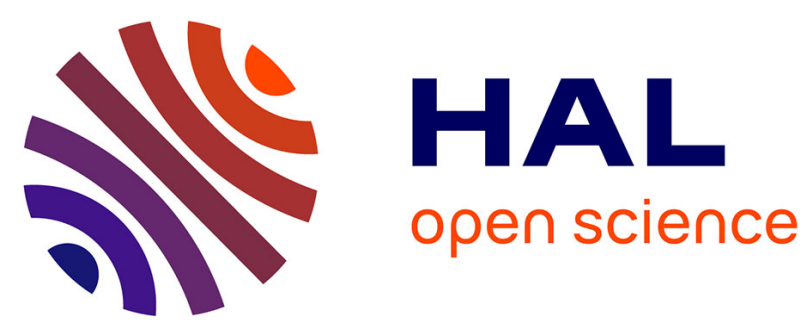

\title{
L'installation de fréquences du laboratoire de spectroscopie hertzienne de la Faculté des Sciences de Lille
}

\author{
R. Wertheimer, J. Bellet, F. Caille, Julien Carlier, J. Messelyn, A. Specq
}

\section{- To cite this version:}

R. Wertheimer, J. Bellet, F. Caille, Julien Carlier, J. Messelyn, et al.. L'installation de fréquences du laboratoire de spectroscopie hertzienne de la Faculté des Sciences de Lille. J. Phys. Phys. Appl., 1963, 24 (S3), pp.9-14. 10.1051/jphysap:019630024030900 . jpa-00212916

\section{HAL Id: jpa-00212916 https://hal.science/jpa-00212916}

Submitted on 1 Jan 1963

HAL is a multi-disciplinary open access archive for the deposit and dissemination of scientific research documents, whether they are published or not. The documents may come from teaching and research institutions in France or abroad, or from public or private research centers.
L'archive ouverte pluridisciplinaire HAL, est destinée au dépôt et à la diffusion de documents scientifiques de niveau recherche, publiés ou non, émanant des établissements d'enseignement et de recherche français ou étrangers, des laboratoires publics ou privés. 


\title{
L 'INSTALLATION DE FRÉQUENCES DU LABORATOIRE DE SPECTROSCOPIE HERTZIENNE DE LA FACULTÉ DES SCIENCES DE LILLE
}

\author{
Par R. WERTHEIMER, J. BELLET, F. CAILLE, J. CARLIER, \\ J. MESSELYN et A. SPECQ, \\ Institut de Physique, Lille.
}

\begin{abstract}
Résumé. - On donne la description d'une installation de fréquences étalon pilotées par un oscillateur à quartz, du type Butler, fonctionnant sur $5 \mathrm{MHz}$. On indique également deux méthodes de mesure de la fréquence de l'oscillateur pilote qui correspondent à des degrés de précision différents.
\end{abstract}

Abstract. - This paper gives the description of $5 \mathrm{Mc}$; quartz crystal controlled frequency standards for microwave spectroscopy, and the two methods of measurement of the absolute frequency of the quartz crystal. These two methods differ, by their degree of accuracy.

Introduction. - Une installation de spectroscopie hertzienne utilisée pour l'étude des spectres des gaz et des vapeurs, comprend nécessairement un générateur de fréquences stables et un équipement pour la mesure absolue de ces fréquences.

L'installation dont nous disposons à Lille est tout à fait classique ; elle a été réalisée par des candidats au Diplôme d'Études Supérieures et nous avons pensé que sa description pourrait être de quelque utilité.

Générateurs de fréquences stables. - La base de cette partie est un oscillateur à quartz thermostaté [1]. Il est constitué par un montage du type Butler où l'on utilise la résonance série d'un quartz pour commander la réaction d'un oscillateur constitué par une double triode à forte pente. Le montage entre cathodes permet d'utiliser des quartz de bonne qualité en réduisant l'amortissement.

La fréquence de ce pilote a été choisie en fonction de l'usage auquel nous le destinons. Nous désirons en effet des fréquences stables dans la gamme qui s'étend de $18 \mathrm{GHz}$ à $36 \mathrm{GHz}$. Il est bien connu que les multiplications successives de fréquences constituent autant de sources de modulations par le bruit et les ronflements résiduels qui parviennent en particulier des alimentations des tubes électroniques. Ceci conduirait donc à choisir un pilote de fréquence élevée ce qui risque alors de reporter la difficulté sur le problème de la mesure absolue de sa fréquence.

A ces deux conditions techniques il faut ajouter que l'on trouve de très bons cristaux pour les fréquences qui correspondent à des émissions étalons. Toutes ces raisons nous ont amenés à choisir un quartz AT sous vide fonctionnant autour de $5 \mathrm{MHz}$. Le tube qui contient le quartz se trouve dans une enceinte thermiquement isolée qui est constituée par un Dewar chauffé par un ensemble de 4 résis- tances disposées en pont de Wheatstone. Deux résistances sont en nickel et servent de thermomètres, les deux autres sont en évanohm et ont au contraire un très faible coefficient de température. Cet ensemble de résistances, bobinées sur mandrin de laiton, convenablement isolé, est alimenté suivant une des diagonales du pont par l'étage de puissance d'un amplificateur basse fréquence dont l'entrée est branchée sur l'autre diagonale. En choisissant

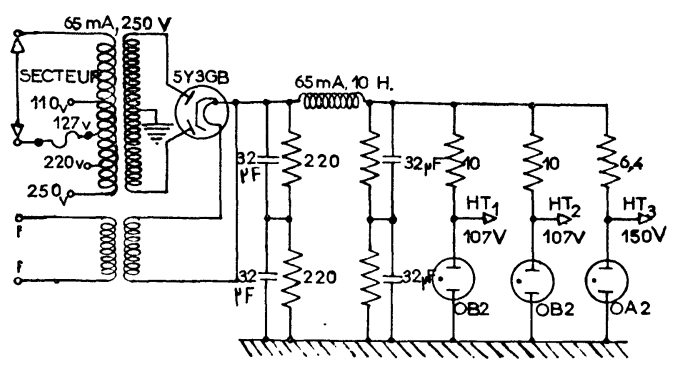

alimentation StABILISEE

DE L'OSCILLATEUR

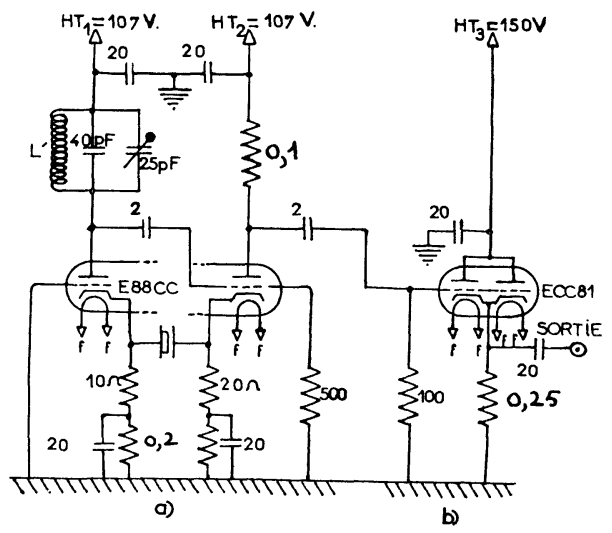

FIG. 1. - Oscillateur à quartz. 
convenablement le branchement on réalise ainsi un servomécanisme qui maintient dans l'enceinte une température fixée par le déséquilibre du pont de Wheatstone. Il suffit de faire varier une des résistances $\mathrm{du}$ pont pour régler la température d'équilibre de l'enceinte. On choisit évidemment le point qui correspond au minimum de dérive thermique du quartz. Pour le cristal que nous utilisons ce point se situe au voisinage de $80^{\circ} \mathrm{C}$.

La figure 1 donne le schéma complet de l'oscillateur à quartz; la figure 2 représente celui du thermostat.

Un ensemble de multiplicateurs à tubes électroniques [2] permet d'obtenir de l'énergie à des fréquences multiples de celle du pilote.

La figure 3 donne des schémas des circuits qui fournissent les fréquences suivantes : $60 \mathrm{MHz}$, $90 \mathrm{MHz}$ et $810 \mathrm{MHz}$.

La puissance délivrée sur $810 \mathrm{MHz}$ est suffisante pour donner environ $2 \mathrm{~mA}$ de courant détecté sur un redresseur de type I N 26 monté, soit dans un guide RG $53 \mathrm{U}$, soit dans un guide RG 96 U. Ces deux guides correspondent respectivement aux bandes des $1,25 \mathrm{~cm}$ et des $8,6 \mathrm{~mm}$ de longueur d'onde. Ce cristal sert à la fois de multiplicateur et de mélangeur c'est-à-dire que l'on y injecte du $810 \mathrm{MHz}$ et que l'on en extrait le battement entre l'harmonique de cette fréquence qui se trouve le plus proche de l'hyperfréquence que l'on veut mesurer et celle-ci. Pour que le système fonctionne correctement il suffit de régler l'hyperfréquence à un niveau suffisamment bas pour ne pas gèner la production des harmoniques du $810 \mathrm{MHz}$.
Ce dispositif nous a permis d'effectuer des mesures jusqu'au voisinage de $36,5 \mathrm{GHz}$.

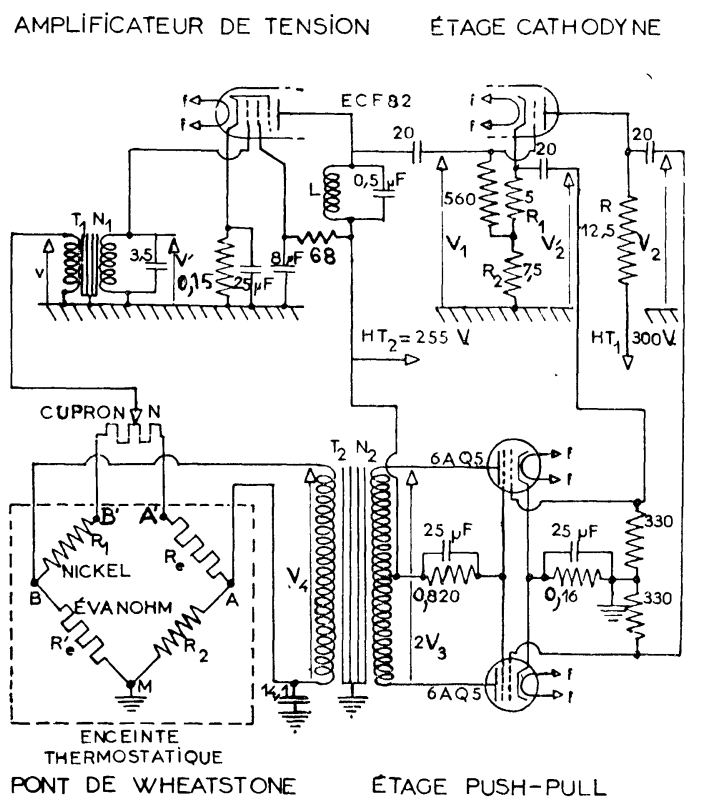

FIG. 2.

L'expérience montre que le $810 \mathrm{MHz}$ obtenu est suffisamment modulé par le $270 \mathrm{MHz}$ qui sert à l'obtenir, de sorte que l'on dispose d'une échelle de fréquences dont l'intervalle est de $270 \mathrm{MHz}$. Les multiplicateurs ont été construits sans faire

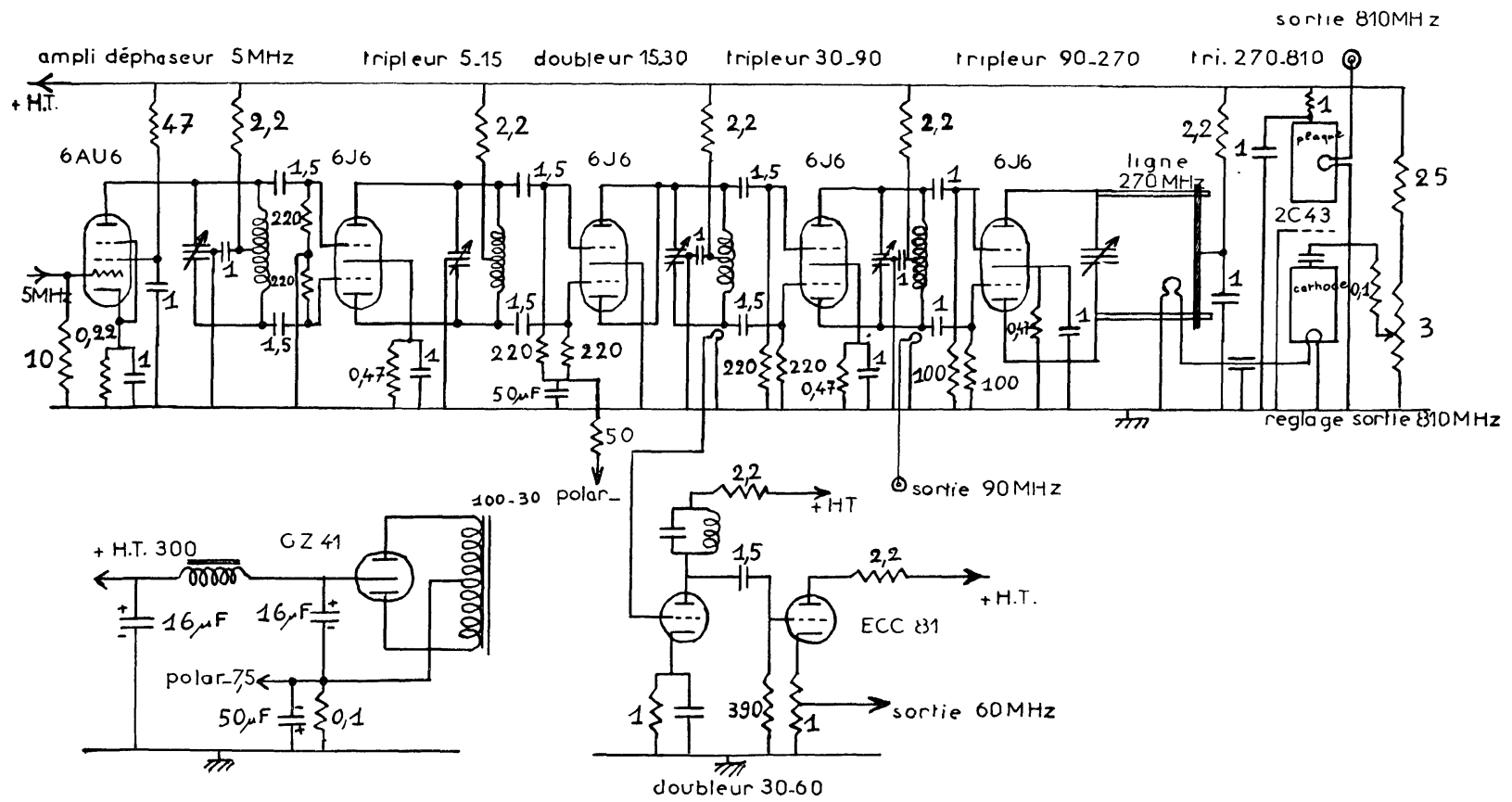

Fig. 3. - Schéma de la chaîne de multiplication. 
appel aux réactances non linéaires à semi-conducteurs qui étaient alors assez peu répandues. Avec de tels dispositifs on devrait obtenir des multiplicateurs dans lesquels s'introduit un minimum de défauts. En effet ils n'utilisent que des tensions continues de polarisation qui ne débitent pas et qui sont donc beaucoup plus faciles à filtrer, et, par prin- cipe, ils sont en outre exempts d'effet de grenaille.

Le groupe des fréquences multiples est complété par trois générateurs de sous-multiples de la fréquence du pilote [3]. Ces générateurs sont tous du type diviseurs à réaction et ils délivrent respectivement $1 \mathrm{MHz}$, du $100 \mathrm{kHz}$ et du $10 \mathrm{kHz}$. La figure 4 donne le schéma de l'ensemble.
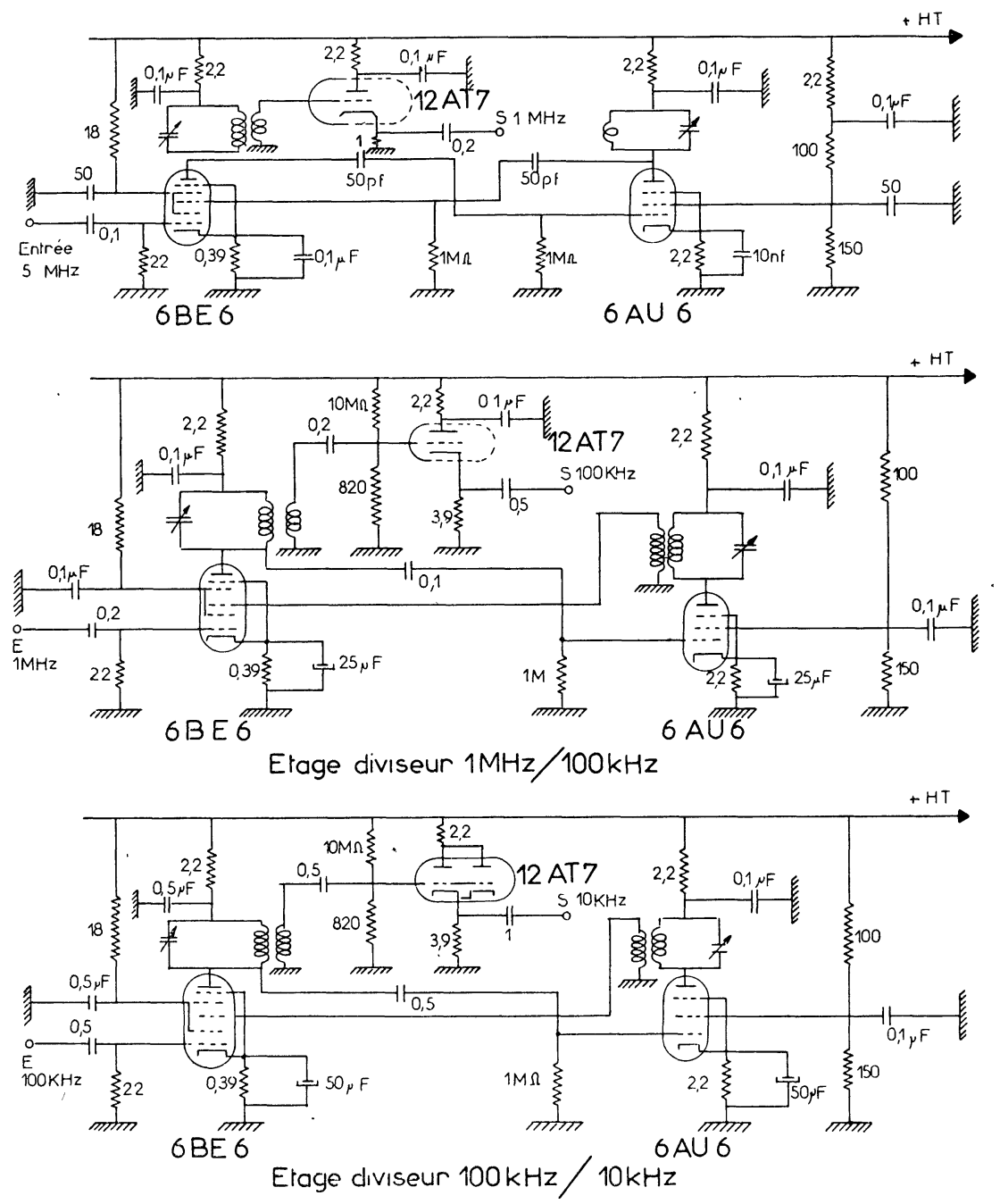

FIG. 4.

Mesure de la fréquence du pilote. - Il s'agit évidemment de mesurer la valeur absolue de la fréquence du pilote ; l'expérience montre que les mesures ordinaires de spectroscopie hertzienne se font avec une erreur relative qui est de l'ordre de $10^{-6}$; il faut donc connaître la fréquence de l'étalon avec une erreur relative de l'ordre de $10^{-7}$. Nous avons utilisé deux méthodes différentes:

a) La première de ces méthodes [4] consiste à comparer la fréquence du pilote à celle d'une des émissions étalon; c'est en partie pour cela que nous avons choisi $5 \mathrm{MHz}$ pour la fréquence du pilote car nous recevons convenablement l'émis- sion diffusée sur cette valeur depuis Rugby sous l'indicatif M. S. F.

Pour appliquer cette méthode il est commode d'avoir un pilote légèrement décalé par rapport à la fréquence nominale, de manière à obtenir un battement basse fréquence à la sortie d'un récepteur réglé sur $5 \mathrm{MHz}$; celui-ci est attaqué simultanément par l'émission étalon arrivant sur l'antenne, et par le rayonnement émis par le pilote. Il ne reste plus alors qu'à mesurer la période du battement avec une précision raisonnable. Pour fixer les idées, si le battement est à $500 \mathrm{~Hz}$, une erreur relative de $10^{-3}$ donne une précision absolue 
de $0,5 \mathrm{~Hz}$, ce qui se traduit par une précision relative de $10^{-7}$ sur la fréquence du pilote.

Malheureusement le battement obtenu n'est pas directement mesurable car son amplitude se ressent du fading qui affecte les ondes en provenance de Rugby. C'est d'ailleurs ce phénomène qui limite les possibilités de cette méthode car son existence est liée à une modification continuelle des différents chemins suivis par les ondes pour venir de l'émetteur au récepteur.

La variation de l'amplitude reçue résulte donc de la combinaison des ondes qui ont suivi des chemins différents et dont la différence de phase présente un caractère aléatoire. C'est cette modulation de phase qui introduit l'imprécision ; la variation d'amplitude est un effet dont l'importance peut être facilement réduite, car dans tous les cas où il s'agit de réaliser une mesure sur un phénomène fluctuant, on effectue la mesure sur un phénomène stable dont les propriétés sont aussi voisines que possible de la moyenne des phénomènes aléatoires. Comme il s'agit ici d'une mesure de période il suffit donc de l'effectuer sur un générateur basse fréquence dont la période est réglée sur celle du battement.

Nous avons construit à cet effet un oscillateur à résistance capacité qui couvre la bande $240 \mathrm{~Hz}$ $310 \mathrm{~Hz}$ en 4 gammes, ce qui permet un réglage fin.

Ce générateur dont le schéma est donné figure 5 délivre deux tensions en quadrature qui permettent

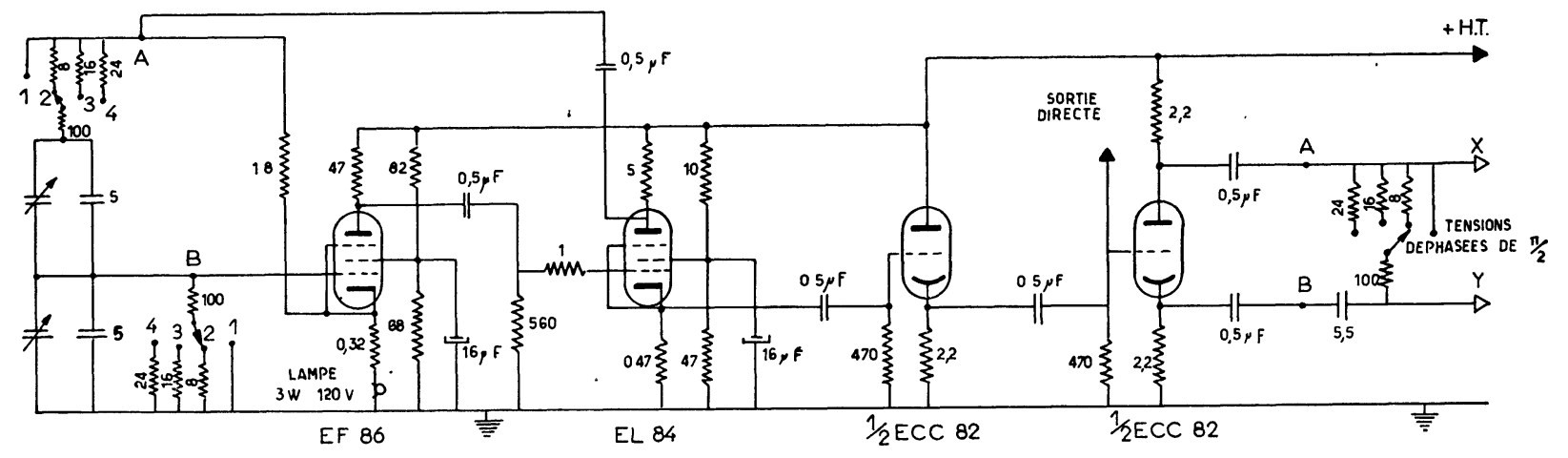

Fig. 5.

d'obtenir le balayage circulaire d'un oscillographe cathodique dont le Whenelt est modulé par le battement à mesurer. Lorsque la fréquence de l'oscillateur BF coïncide avec celle du battement on obtient alors un demi-cercle illuminé qui est fixe sur l'écran de l'oscillographe ; il ne reste plus alors qu'à mesurer la période du générateur au compteur électronique. Il est en effet bien connu, que pour les fréquences basses, le compteur donne une meilleure précision dans la mesure de la période que dans celle de la fréquence. Cette méthode permet une mesure rapide; il suffit d'une faible rotation du demi-cercle lumineux pendant quelques secondes pour que l'on puisse apprécier le synchronisme du battement et du générateur avec une précision suffisante pour en déduire la fréquence $d u$ pilote avec une erreur relative de $10^{-7}$. La mesure au compteur électronique introduit ici une erreur qui reste toujours négligeable.

En fait, le demi-cercle lumineux fluctue presque sans arrêt et il ne reste plus qu'à apprécier l'existence d'un déplacement lent superposé à des fluctuations ; c'est la faiblesse du procédé qui donne des résultats convenables en dehors des périodes troubles du lever et du coucher du soleil.

b) La seconde méthode ne dépend pas de la fréquence de l'émission étalon; elle utilise les émis- sions des signaux horaires ; c'est une méthode de coïncidence.

Les signaux horaires radiodiffusés par les stations à ondes courtes sont constitués pour l'essentiel par des impulsions de 5 millisecondes. Un programme horaire qui varie d'une station à l'autre permet de repérer le temps et ne joue aucun rôle dans la mesure qui nous intéresse.

Celle-ci consiste à mesurer le temps qui s'écoule entre deux coïncidences successives du signal étalon avec un signal à la seconde obtenu à partir du pilote local. Désignons par $x$ la fréquence de celui-ci exprimée en $\mathrm{MHz}$.

Un dispositif électronique [5] permet d'obtenir un signal qui se reproduit avec une fréquence de $x / 5$ (pratiquement $1 \mathrm{~Hz}$ ) ou une période $T=5 \mathrm{~s} / x$ et dont la position peut être réglée par bonds de $0,05 \mathrm{~s} / x$ (pratiquement $0,01 \mathrm{~s}$ ). Ce dispositif comprend un diviseur apériodique à tubes à vide alimenté par le $10 \mathrm{kHz}$ dérivé de l'étalon ; il fournit deux séries d'impulsions. La première est constituée par des signaux espacés de 5 s/10 $x$ (pratiquement $0,1 \mathrm{~s}$ ) qui alimentent un dékatron dont toutes les cathodes sont accessibles. On obtient ainsi des impulsions à la fréquence $x / 5$ dont la position est réglable par bonds de $5 \mathrm{~s} / 10 x$; ces impulsions commandent l'ouverture d'une porte de 
largeur convenable mais fixe et dont la position peut être réglée par bonds de 5 s/100 $x$. En utilisant les 10 cathodes du dékatron il suffit de dix positions de la porte pour que l'on puisse choisir une des cent impulsions de la deuxième série qui sont espacées de $5 \mathrm{~s} / 100 x$.

Ce dispositif permet de résoudre le problème en évitant l'incertitude sur le signal fourni par le dékatron qui est un tube à gaz.

La figure 6 donne le schéma du diviseur apériodique à décades qui est précédé par un étage de mise en forme et la figure 7 donne le schéma des circuits qui permettent de sélectionner une des impulsions fournies par le diviseur toutes les
$5 \mathrm{~s} / 100 x$. Dans le cas du pilote dont nous disposons, sa fréquence est légèrement inférieure à $5 \mathrm{MHz}$, de sorte que si l'on compte le nombre $n$ d'impulsions de fréquence $x / 5$ fournies entre $p$ coïncidences, on a évidemment :

$$
(n+p) 1 \mathrm{~s}=n T=5 n / x
$$

d'où

$$
x \mathrm{MHz}=5 n /(n+p) .
$$

Pratiquement on détecte la coïncidence grace à un oscillographe dont on déclenche le balayage à l'aide du signal de fréquence $x / 5$.

L'amplificateur vertical de l'oscillographe est

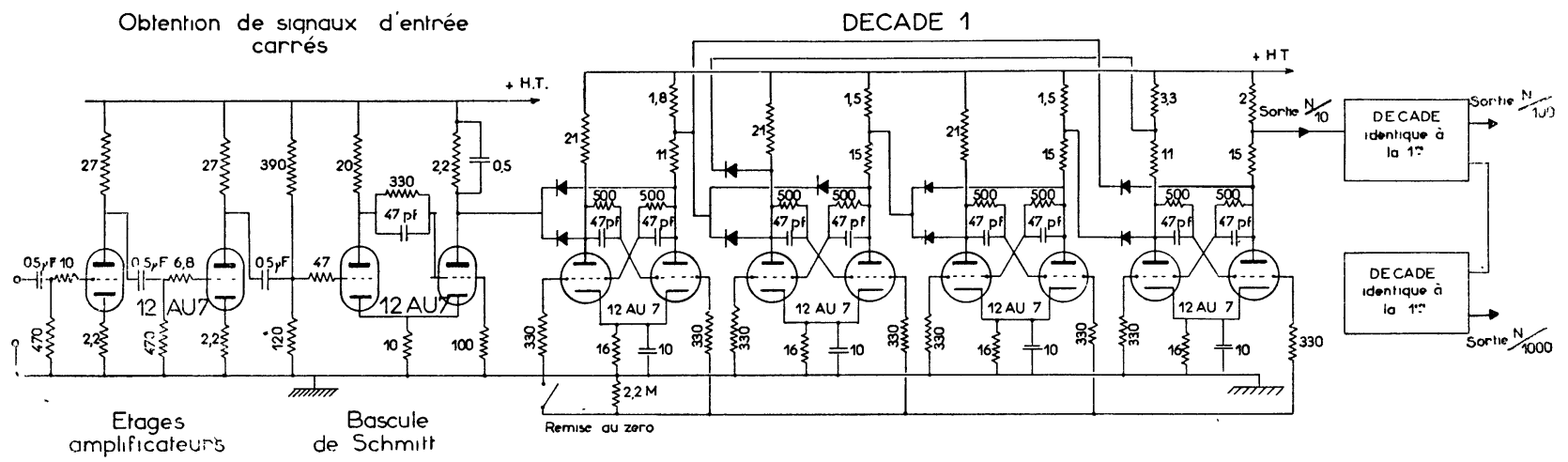

FIG. 6.
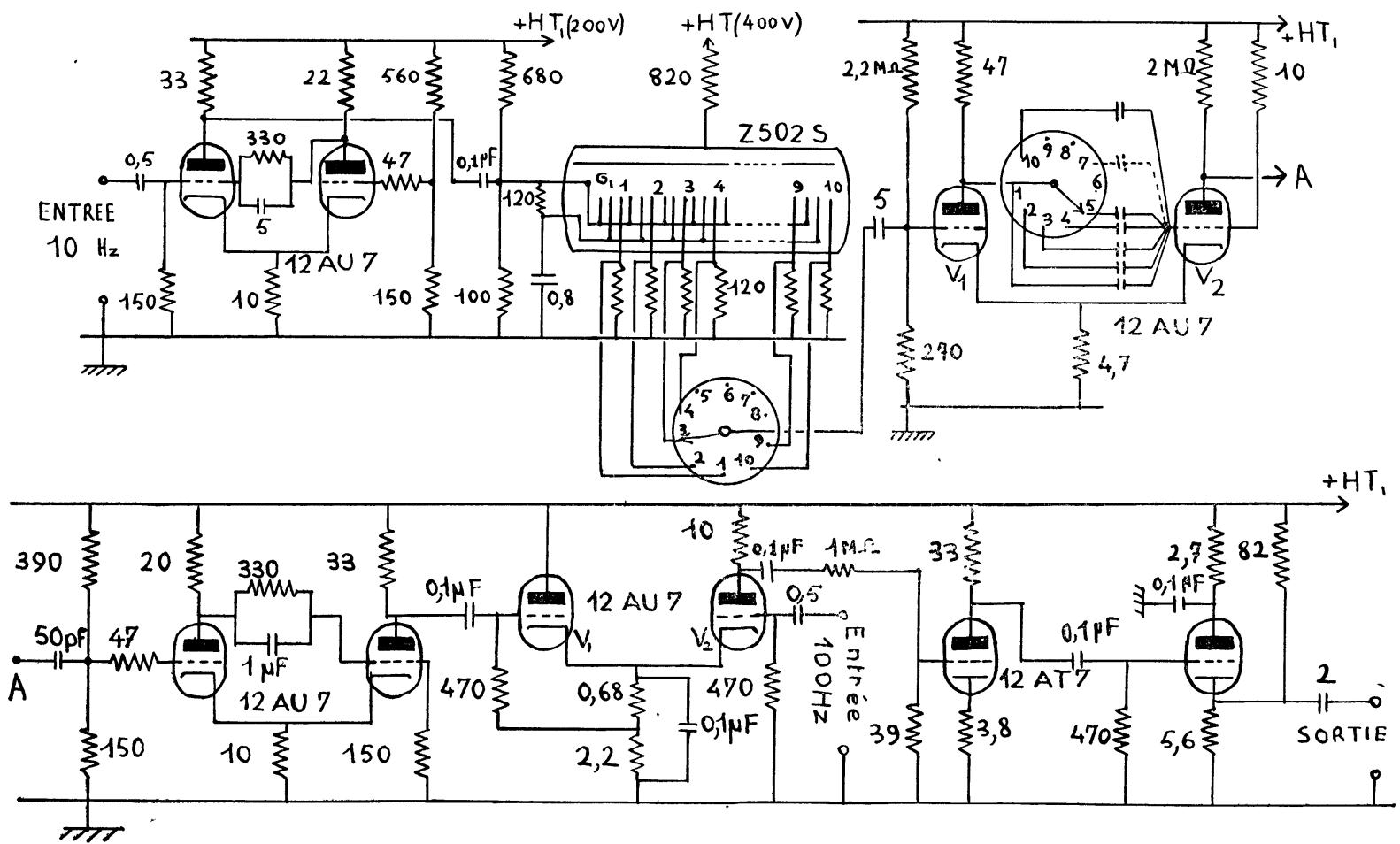

FIG. 7. 
alimenté par le signal de sortie d'un récepteur réglé sur l'émission de signaux horaires et il ne reste plus qu'à ajuster la position du déclenchement pour que le signal reçu soit visible sur l'écran.

Dans cette méthode il existe deux sources d'erreur; la première est d'ordre expérimental et concerne la difficulté d'apprécier les coïncidences extrêmes ; la seconde provient de la propagation des ondes qui s'accompagne de fluctuations dans la durée du trajet. Ces causes d'erreur provoquent toutes deux une erreur $\Delta n$ sur le nombre $n$, ce qui entraîne une erreur $\Delta x$ sur $x$ qui est donnée par :

$$
\Delta x / x=p \Delta n / n(n+p) \simeq p \Delta n / n^{2}
$$

Pour obtenir une précision élevée il faut donc avoir un nombre $n$ assez grand, ce qui nécessite une durée de mesure de l'ordre de la journée si l'on veut une précision relative de $10^{-8}$ sur la fréquence du pilote.

Pour réaliser une mesure précise et plus rapide nous songeons à utiliser les émissions en ondes myriamétriques en provenance des États-Unis et dont certaines peuvent être captées facilement en Europe.

Pour terminer cette revue des travaux qui ont été réalisés, à Lille, pour construire l'ensemble de mesure du Laboratoire de Spectroscopie Hertzienne, nous voudrions exprimer notre reconnaissance à M. Decaux, Ingénieur en chef des Télécommunications qui dirige le département fréquences du Centre National des Télécommunications, à son adjoint M. Gabry et à tout le personnel du département pour les conseils et l'aide qu'ils nous ont apportés.

Il nous est agréable de remercier tout le personnel du service de Physique Générale de la Faculté pour sa collaboration dans la réalisation pratique de cet ensemble.

Manuscrit reçu le 11 juillet 1962.

\section{BIBLIOGRAPHIE}

[1] Carlier (J.), D. E. S., Lille, 1961.

[2] Caille (F.), D. E. S., Lille, 1961.

[3] Messelyn, D. E. S., Lille, 1961.
[4] Bellet (J.), D. E. S., Lille, 1961.

[5] Spece (A.), D. E. S., Lille, 1962. 